\title{
NP-completeness of list coloring and precoloring extension on the edges of planar graphs
}

\author{
Dániel Marx*
}

17th October 2004

\begin{abstract}
In the edge precoloring extension problem we are given a graph with some of the edges having a preassigned color and it has to be decided whether this coloring can be extended to a proper $k$-edge-coloring of the graph. In list edge coloring every edge has a list of admissible colors, and the question is whether there is a proper edge coloring where every edge receives a color from its list. We show that both problems are NP-complete on (a) planar 3-regular bipartite graphs, (b) bipartite outerplanar graphs, and (c) bipartite series-parallel graphs. This improves previous results of Easton and Parker [6], and Fiala [8].
\end{abstract}

\section{Introduction}

In graph vertex coloring we have to assign colors to the vertices such that neighboring vertices receive different colors. Starting with [7] and [28], a generalization of coloring was investigated: in the list coloring problem each vertex can receive a color only from its prescribed list of admissible colors. In the precoloring extension problem a subset $W$ of the vertices have preassigned colors and we have to extend this precoloring to a proper coloring of the whole graph, using only colors from a given color set $C$. It can be viewed as a special case of list coloring: the list of a precolored vertex consists of a single color, while the list of every other vertex is $C$. A thorough survey on list coloring, precoloring extension, and list chromatic number can be found in $[26,1,12,13]$.

In this paper we consider the edge coloring version of list coloring and precoloring extension. Colbourn [3] has shown that edge precoloring extension is NP-complete for complete bipartite graphs. Easton and Parker [6], and independently Fiala [8] have shown that the problem is NP-complete for 3-regular bipartite graphs. In Section 2 we strengthen this result by showing that the problem remains NP-complete for planar 3-regular bipartite graphs. The main

${ }^{*}$ Dept. of Computer Science and Information Theory, Budapest University of Technology and Economics, Budapest H-1521, Hungary (dmarx@cs.bme.hu). Research supported by OTKA grants 44733, 42559 and 42706. 
difficulty of the proof is to find gadgets that are planar and 3-regular at the same time. As the first step of the proof, we show that list edge coloring is also NP-complete on the same class of graphs.

The unrestricted version of edge coloring is known to be NP-complete [11, 18]. However, the problem is easy for bipartite graphs: by König's Line Coloring Theorem [16], every bipartite graph with maximum degree $\Delta$ can be edge colored with $\Delta$ colors. Vizing [27] has show that every planar graph with maximum degree $\Delta \geq 8$ can be edge colored with $\Delta$ colors, and conjectured that the same holds for every $\Delta \geq 6$. The case $\Delta=7$ was proved by Sanders and Zhao [25]. For $\Delta=3,4,5$ there are planar graphs with maximum degree $\Delta$ that cannot be edge colored with $\Delta$ colors. Hence edge coloring planar graphs is easy for $\Delta \geq 7$, expected to be easy for $\Delta=6$, and the complexity is open for $\Delta=3,4,5$. (See [30] for an overview of algorithmic results on edge coloring.) Our results show that the more general list edge coloring and edge precoloring extension problems are NP-hard even if the graph is both planar and bipartite.

In $[10,9,15,29]$ the list edge coloring problem is considered for series-parallel graphs, sufficient conditions are given for some special cases. In Section 3 we investigate the computational complexity of the problem. An easy argument shows that precoloring edge extension and list edge coloring can be solved in polynomial-time for bounded degree outerplanar graphs. However, we prove that the problems are NP-complete for outerplanar and series-parallel graphs if the maximum degree can be arbitrary.

Outerplanar and series-parallel graphs have treewidth at most 2, thus the results of Section 3 show that precoloring edge extension and list edge coloring are NP-complete for partial 2-trees. However, both problems can be solved in polynomial time for trees (see [19]). This is somewhat surprising, since there are very few problems that are polynomial-time solvable for trees but NP-hard for partial 2-trees. Usually it is expected that if a dynamic programming approach works for trees, then it can be generalized to partial $k$-trees. A recent example where the problem is easy for trees but NP-hard for partial 2-trees is the edge disjoint paths problem [23].

\section{Planar bipartite graphs}

In this section we prove that the precoloring extension problem is NP-complete on the edges of planar 3-regular bipartite graphs. First we prove that the more general list edge coloring problem is NP-complete for such graphs, then we present a reduction from list coloring to precoloring extension.

The proof is by reduction from the 1-in-3 Satisfiablity problem, which is the following: given a formula in conjunctive normal form, every clause contains exactly 3 literals, decide if a variable assignment exists such that exactly one literal is true in every clause. The 1-in-3 Satisfiability problem remains NPcomplete even with the following restrictions:

Theorem 2.1 ([22]). 1-in-3 Satisfiablity is NP-complete even if 


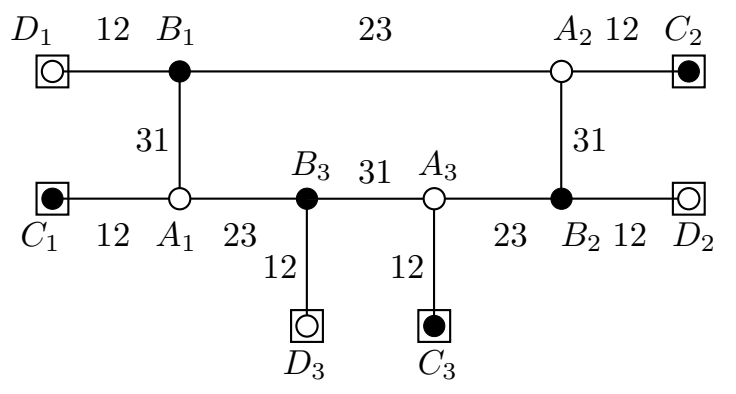

Figure 1: The variable setting gadget. The two numbers on each edge show the list of available colors.

- every variable appears in exactly 3 clauses,

- there is no negation in the formula, and

- the underlying bipartite graph of the formula (where the vertex representing a clause is connected to vertices representing the variables appearing in the clause) is planar.

Theorem 2.2. List edge coloring is NP-complete for planar 3-regular bipartite graphs.

Proof. We construct variable setting gadgets and satisfaction testing gadgets, and connect them in such a way that the resulting graph can be colored if and only if the given formula is satisfiable (in 1-in-3 sense). If the original formula satisfies the requirements of Theorem 2.1, then the resulting graph is planar and 3 -regular.

Figure 1 shows the variable setting gadget. It is easy to verify that it has only two colorings: the coloring that assigns the first (resp. second) color of the list to each edge. Therefore in every coloring of the gadget, the pendant edges receive the same color, either 1 or 2 . The coloring that assigns color 1 to the pendant edges corresponds to setting the variable to "true," and the coloring that assigns 2 to these edges corresponds to "false."

The satisfaction testing gadget is shown on Figure 2. We claim that it has only three colorings, the numbers in the frames are the colors assigned to the edges in the three colorings. Let $\psi$ be a coloring of this gadget. First, let us verify that $\psi\left(A_{i} C_{i}\right)=\psi\left(B_{i} D_{i}\right)$ for $i=1,2,3$ in every coloring $\psi$. For this purpose, it is sufficient to follow the implications of say, $\psi\left(A_{1} C_{1}\right)=1$ and $\psi\left(B_{1} D_{1}\right)=2$, to arrive to a contradiction. The case $i=3$ is somewhat more complicated to verify: $\psi\left(A_{3} C_{3}\right)=1$ and $\psi\left(B_{3} D_{3}\right)=2$ imply $\psi\left(C_{3} D_{3}\right)=\psi\left(E_{3} F_{3}\right)=3, \psi\left(C_{3} E_{3}\right)=2$, $\psi\left(D_{3} F_{3}\right)=1$; edges $D_{3} F_{3}$ and $E_{3} F_{3}$ force $\psi\left(F_{3} E_{2}\right)=2, \psi\left(F_{2} E_{2}\right)=1$; edges $C_{3} E_{3}$ and $E_{3} F_{3}$ force $\psi\left(J_{1} E_{3}\right)=1, \psi\left(J_{1} I_{1}\right)=3, \psi\left(H_{1} J_{1}\right)=2, \psi\left(H_{1} G_{1}\right)=3$, $\psi\left(E_{1} G_{1}\right)=1$, and $\psi\left(G_{1} I_{1}\right)=2$. Now there is no color left for edge $I_{1} F_{2}$, since edges $F_{2} E_{2}, G_{1} I_{1}$, and $J_{1} I_{1}$ use all three colors. Furthermore, it can be shown 


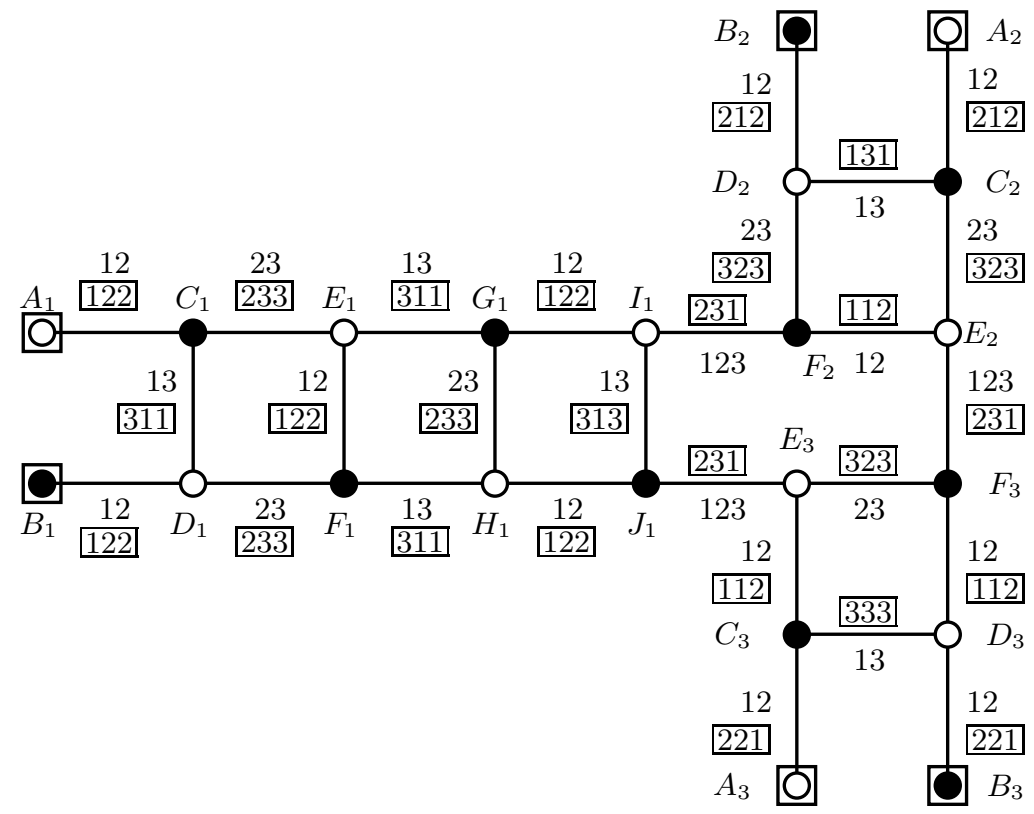

Figure 2: The satisfaction-testing gadget. The numbers on the edges show the list of available colors on the edge. The three numbers in each frame show the three colors assigned to the edge in the three possible colorings of the graph.

that $\psi\left(A_{1} C_{1}\right)=\psi\left(B_{1} D_{1}\right)=1$ implies that $\psi$ is the first coloring defined on Figure 2. Similarly, $\psi\left(A_{2} C_{2}\right)=\psi\left(B_{2} D_{2}\right)=1\left(\right.$ resp. $\left.\psi\left(A_{3} C_{3}\right)=\psi\left(B_{3} D_{3}\right)=1\right)$ implies that $\psi$ is the second (resp. third) coloring. Thus in every coloring of the gadget, exactly one of the pairs $A_{i} C_{i}$ and $B_{i} D_{i}$ is colored with color 1, the others with color 2. A coloring that assigns color 1 to $A_{i} C_{i}$ and $B_{i} D_{i}$ corresponds to a variable assignment where the clause is satisfied by its $i$ th literal.

Figure 3 shows the overview of the construction. Take a copy of the variable setting gadget $G_{x}$ for each variable $x$ and a satisfaction-testing gadget $G_{C}$ for each clause $\mathrm{C}$ of the formula. If $x$ appears in clause $C$, then $G_{x}$ and $G_{C}$ are connected by a pair of edges. Fix a planar embedding of the formula, and fix an ordering of the occurences of each variable in such a way that the clauses of a variable are located in clockwise order around the variable. Similarly, for each clause fix an ordering of the literals in such a way that the variables of a clause are in clockwise order around the clause. Assume that $x$ is the $i$ th variable in clause $C$, and $C$ is the $j$ th clause where $x$ appears, then connect $G_{x}$ and $G_{C}$ by identifying the edges $A_{j} C_{j}$ and $B_{j} D_{j}$ of $G_{x}$ with $A_{i} C_{i}$ and $B_{i} D_{i}$ of $G_{C}$, respectively.

The resulting graph is bipartite since the gadgets are bipartite (Figure 1 and 2 show the two color classes) and when we identified two edges, we only identified vertices that belong to the same color class. Since every variable 


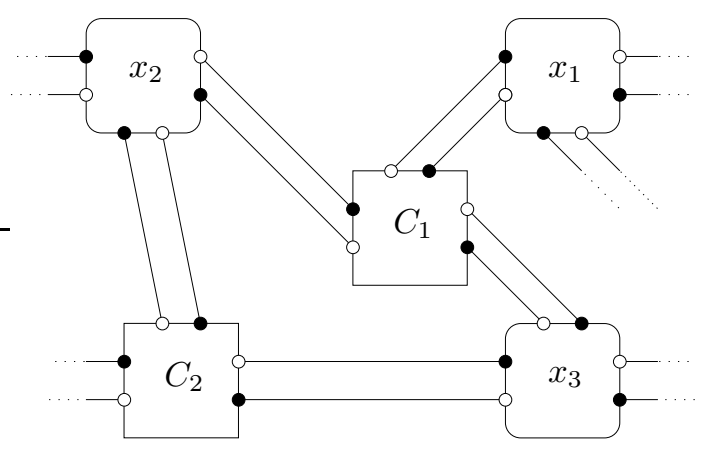

Figure 3: The reduction from 1-in-3 SAT to the list edge coloring problem. We connect the variable setting components and the satisfaction testing components with pairs of edges.

appears in exactly three clauses and every clause has exactly three literals, the resulting graph is 3-regular. The gadgets are planar, and because of the planarity of the formula, the graph can be embedded in the plane such that two pairs of edges do not cross each other. Note that in the variable setting gadget, edge $B_{j} D_{j}$ is in clockwise direction from the edge $A_{j} C_{j}$, while in the satisfaction testing gadget $B_{i} D_{i}$ is in counterclockwise direction from $A_{i} C_{i}$. Thus the two edges connecting a variable setting gadget with a satisfaction testing gadget do not cross each other, as shown on Figure 3. Therefore the resulting graph $\mathrm{G}$ is planar, bipartite and 3-regular.

It is clear from the construction that $G$ has a proper list edge coloring if and only if the formula has a satisfying variable assignment (in 1-in-3 sense). Obviously, the graph can be built in polynomial time, thus we have proved that the problem is NP-complete.

List coloring is reduced to precoloring extension by locally replacing the edges by some partially precolored graphs.

Theorem 2.3. Precoloring extension is NP-complete on the edges of planar 3-regular bipartite graphs.

Proof. We reduce list edge coloring to precoloring extension as follows. By the proof of Theorem 2.2, it can be assumed that only the three colors 1, 2, 3 appear in the lists. If only a single color appears in the list of an edge, then the edge is precolored with that color. If all three colors appear in the list of an edge, then the edge is not precolored. If edge $u v$ has a 2 element list, say color $i$ is not allowed $(1 \leq i \leq 3)$, then we replace $u v$ by the gadget shown on Figure 4 . The edges $x_{1} x_{3}$ and $x_{2} x_{4}$ are precolored with color $i$.

We claim that the list edge coloring problem has a solution in the original graph $G$ if and only if the precoloring in the constructed graph $G^{\prime}$ can be extended to a proper 3-edge-coloring. First, a coloring of $G$ can be used to 


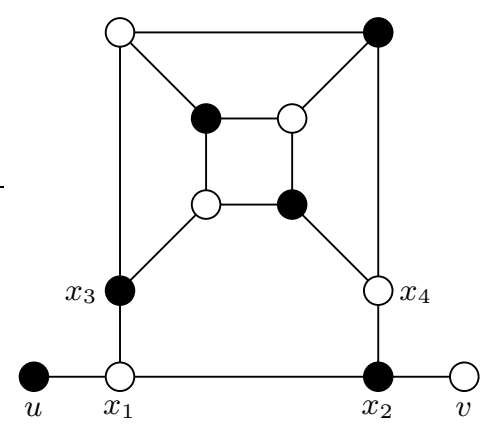

Figure 4: The gadget used to replace those edges that have 2 element lists.

obtain a precoloring extension of $G^{\prime}$ : if edge $u v$ has color $c$, then the gadget corresponding to $u v$ can be colored such that both $u x_{1}$ and $x_{2} v$ receive color $c$. On the other hand, in every 3-edge-coloring of $G^{\prime}$ edges $u x_{1}$ and $x_{2} v$ receive the same color, a color different from the forbidden color $i$ of edge $u v$ : since both $x_{1} x_{3}$ and $x_{2} x_{4}$ are precolored to $i$, only two colors are available for the edges $u x_{1}, x_{1} x_{2}, x_{2} v$. Therefore a precoloring extension of $G^{\prime}$ determines a list coloring of $G$.

\section{Outerplanar graphs}

A graph is outerplanar if it has a planar embedding such that all the vertices lie on the exterior face. A graph is series-parallel if it can be created from $K_{2}$ by repeatedly duplicating and subdividing the edges. If an outerplanar graph is 2connected, then it is series-parallel. Some authors give a more general definition of series-parallel graphs by allowing to start from an arbitrary tree instead of a $K_{2}$. We are giving complexity results here, thus using the more restrictive definition makes our results stronger.

Edge precoloring extension can be solved in linear time for bounded degree outerplanar and series-parallel graphs as follows. It is well-known that these graphs have treewidth at most 2 . We show that if both the treewidth and the maximum degree of a graph are bounded by a constant, then precoloring extension can be solved in linear time. First, if the graph has maximum degree $d$, and the number of available colors is greater than $2(d-1)$, then the problem becomes trivial: every edge can receive a color that is not used on the at most $2(d-1)$ edges adjacent to it. Therefore it can be assumed that there are at most $2(d-1)$ available colors. If a graph has treewidth at most $w$ and maximum degree $d$, then the treewidth of its line graph is at most $(w+1) d-1$ (see $[2$, Lemma 32]). Edge precoloring extension is the same as precoloring extension in the line graph. By [14], precoloring extension can be solved in linear time if both the treewidth and the number of available colors are constant. (This result also follows from the fact that precoloring extension with a fixed number 
of colors can be expressed in monadic second order logic, see [4, 17].) A similar argument works in the case of list edge coloring.

Thus Theorem 2.3 cannot be strengthened to outerplanar graphs. However, if we drop 3-regularity, then the problem remains NP-complete for bipartite outerplanar graphs and series-parallel graphs. In the proof we follow the same path as in Section 2: first it is shown that list coloring is NP-complete with the given restrictions, then list coloring is reduced to precoloring extension.

Theorem 3.1. List edge coloring is NP-complete for bipartite outerplanar graphs.

Proof. The proof is by reduction from 3SAT. Given a formula $\phi$ in conjunctive normal form with $n$ variables and $m$ clauses, we construct an instance of the list edge coloring problem in such a way that the graph can be colored if and only if $\phi$ is satisfiable.

We assume that every variable occurs exactly twice positively and exactly twice negated in $\phi$, and every clause contains exactly 3 literals. This can be achieved as follows. It is well-known that 3SAT remains NP-complete if every variable occurs exactly twice positively, exactly once negated, and every clause contains two or three literals (see e.g., [24, Section 9]). Let us assume that the number of variables is even, if not, then duplicate every variable and every clause. Let $x_{1}, x_{2}, \ldots, x_{t}$ be the variables of $\phi$. We add $t / 2$ new variables $y_{1}, y_{2}, \ldots, y_{\frac{t}{2}}$ and $t$ new clauses $\left(\bar{x}_{1} \vee y_{1} \vee \bar{y}_{1}\right),\left(\bar{x}_{2} \vee y_{1} \vee \bar{y}_{1}\right),\left(\bar{x}_{3} \vee y_{2} \vee \bar{y}_{2}\right)$, $\left(\bar{x}_{4} \vee y_{2} \vee \bar{y}_{2}\right), \ldots,\left(\bar{x}_{t-1} \vee y_{\frac{t}{2}} \vee \bar{y}_{\frac{t}{2}}\right),\left(\bar{x}_{t} \vee y_{\frac{t}{2}} \vee \bar{y}_{\frac{t}{2}}\right)$ to the formula. Now every variable occurs exactly twice positively and twice negated. These new clauses are satisfied in every variable assignment, hence the new formula is satisfiable if and only if the original is satisfiable. Furthermore, if there is a clause $(x \vee y)$ containing only two literals, then add a new variable $z$, and replace this clause with $(x \vee z \vee z) \wedge(\bar{z} \vee \bar{z} \vee y)$. It is easy to see that this transformation does not change the satisfiability of the formula.

The set of colors $C$ contains $4 n$ colors: there is one color corresponding to each occurence of a variable. For $1 \leq i \leq n$, color $4 i$ corresponds to the first positive occurence of $x_{i}$, color $4 i-1$ corresponds to the second positive occurence of $x_{i}$, color $4 i-2$ corresponds to the first negated occurence of $x_{i}$, and color $4 i-3$ corresponds to the second negated occurence of $x_{i}$.

We construct the list edge coloring instance as follows. Let $v$ be a vertex. For every $1 \leq i \leq n$, attach 5 new vertices $a_{i}, b_{i}, c_{i}, d_{i}, e_{i}$ to $v$ as shown on Figure 5 . These edges correspond to variable $x_{i}$. The lists are as shown on the figure. For every $1 \leq j \leq m$, a new vertex $u_{j}$ is attached to $v$. The list of $v u_{j}$ contains three colors: the colors that correspond to the three literals in the $j$ th clause of $\phi$.

Given a satisfying assignment of $\phi$, we construct a coloring $\psi$ of $G$. If variable $x_{i}$ is true, then the 6 edges corresponding to $x_{i}$ receive the first color from their lists. If $x_{i}$ is false, then the edges receive the second color from the lists. Notice that with this coloring the colors used on edges incident to $v$ are exactly those colors that correspond to the false literals in the assignment. By assumption, the assignment satisfies every clause of the formula, every clause contains at 


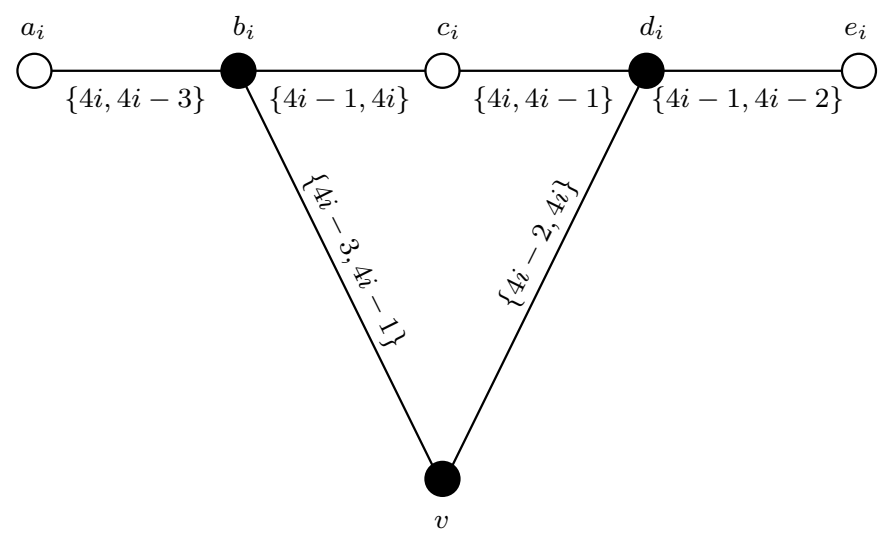

Figure 5: The edges corresponding to variable $x_{i}$ of the formula.

least one true literal, hence the list of every edge $v u_{j}$ contains at least one color not used on $v$. Therefore we can extend $\psi$ to the edges $v u_{j}$, and the graph can be colored.

To prove the other direction assume that there is a coloring $\psi$ of $G$. We show that for every $1 \leq i \leq n$, either

- $\psi\left(v b_{i}\right)=4 i-3$ and $\psi\left(v d_{i}\right)=4 i-2$, or

- $\psi\left(v b_{i}\right)=4 i-1$ and $\psi\left(v d_{i}\right)=4 i$ hold.

If $\psi\left(v b_{i}\right)=4 i-3$, then $\psi\left(a_{i} b_{i}\right)=4 i \Rightarrow \psi\left(b_{i} c_{i}\right)=4 i-1 \Rightarrow \psi\left(c_{i} d_{i}\right)=4 i \Rightarrow$ $\psi\left(v d_{i}\right)=4 i-2$. A similar argument shows that if $\psi\left(v b_{i}\right)=4 i-1$, then $\psi\left(v d_{i}\right)=4 i$ follows. We set variable $x_{i}$ to true in the first case, and to false in the second case. This yields a satisfying variable assignment of $\phi$ : if the $j$ th clause is not satisfied, then all of its literals are false, which implies that the colors corresponding to these literals appear at $v$. However, this means that the three colors in the list of $v u_{j}$ are already used at $v$, therefore edge $v u_{j}$ cannot receive any color, contradicting the assumption that $\psi$ is a list coloring of $G$.

Theorem 3.2. Precoloring extension is NP-complete on the edges of bipartite outerplanar graphs.

Proof. The proof is by reduction from the list edge coloring problem. Consider a bipartite outerplanar graph constructed by the reduction of Theorem 3.1. Notice that every edge lies on the outer face. Let the color set $C$ be the union of the lists. If the list of an edge $x y$ contains 2 colors, then this edge is replaced as follows. We add two new vertices $x^{\prime}, y^{\prime}$, and 3 new edges $x x^{\prime}, x^{\prime} y^{\prime}, y^{\prime} y$. Furthermore, we attach $|C|-2$ new edges $x^{\prime} x_{1}^{\prime}, \ldots, x^{\prime} x_{|C|-2}^{\prime}$ to vertex $x^{\prime}$, and another $|C|-2$ new edges $y^{\prime} y_{1}^{\prime}, \ldots, y^{\prime} y_{|C|-2}^{\prime}$ to $y^{\prime}$. Denote by $c_{1}, \ldots, c_{|C|-2}$ the $|C|-2$ colors in $C$ that do not appear in the list of $x y$. The edges $x^{\prime} x_{i}^{\prime}$ and $y^{\prime} y_{i}^{\prime}$ are precolored with color $c_{i}$. These precolored edges ensure that in every edge 
coloring $x x^{\prime}, x^{\prime} y^{\prime}, y^{\prime} y$ receive a color from the list of $x y$, which implies that the colors of $x x^{\prime}$ and $y^{\prime} y$ are the same. Therefore edges $x x^{\prime}$ and $y y^{\prime}$ effectively act as a single edge with the same list as $x y$. Moreover, since $x y$ is on the outer face, the graph remains outerplanar and bipartite.

Consider and edge $v u_{j}$ having list size 3 . Here we use the fact that $u_{j}$ has degree 1 . We attach $|C|-3$ new edges $u_{j} u_{j, 1}, \ldots, u_{j} u_{j,|C|-3}$ to $u_{j}$, and precolor them with the colors not in the list of $v u_{j}$. It is clear that now edge $v u_{j}$ has to receive a color from the list of $v u_{j}$. Therefore we have constructed an instance of the edge precoloring extension problem that has a solution if and only if the list edge coloring problem has a solution.

Corollary 3.3. Precoloring extension is NP-complete on the edges of bipartite series-parallel graphs.

Proof. We show how to make a bipartite outerplanar graph 2-connected without changing the solvability of the edge precoloring extension instance. Together with Theorem 3.2 and the fact that 2-connected outerplanar graphs are seriesparallel, this proves that edge precoloring extension is NP-complete for seriesparallel graphs.

The graph is outerplanar, hence by traversing the boundary of the outer face we visit every vertex at least once. Since there can be cutvertices in the graph, there might be vertices that are visited more than once. Let $v_{0}, v_{1}$, $\ldots, v_{n-1}$ the order of vertices as they are first encountered while traversing the boundary of the outer face. We add new vertices and edges to the graph to make it Hamiltonian, and therefore 2-connected. If $v_{i}$ and $v_{i+1}$ (indices are taken modulo $n$ ) belong to the same bipartition class, then add a new vertex $w_{i}$ and two new edges $v_{i} w_{i}$ and $w_{i} v_{i+1}$. Edge $v_{i} w_{i}$ is precolored with a new color $\alpha$, and $w_{i} v_{i+1}$ is precolored with a new color $\beta$. If $v_{i}$ and $v_{i+1}$ are in different classes, then add two new vertices $w_{i}^{\prime}, w_{i}^{\prime \prime}$, and three edges $v_{i} w_{i}^{\prime}$ (precolored to $\alpha), w_{i}^{\prime} w_{i}^{\prime \prime}$ (not precolored), $w_{i}^{\prime \prime} v_{i+1}$ (precolored to $\beta$ ). It can be shown that the graph remains outerplanar after these modifications. The two new colors $\alpha$ and $\beta$ appear at every vertex of the original graph, hence they cannot be used for the original edges. Thus the solvability of the instance did not change.

\section{Conclusions}

In this paper we have considered the edge precoloring extension and list edge coloring problems on certain restricted graph classes. First we proved hardness results for list edge coloring, since building gadgets is more convenient in this problem. With simple local replacements, we reduced the list coloring problem to precoloring extension. It turned out that both problems are NP-complete for planar 3-regular bipartite graphs. This result can be useful in showing the NPcompleteness of other planar graph problems. For example, this result is used in [5] to show the NP-hardness of weighted edge coloring of planar 3-regular 
bipartite graphs, and in [20] to show that minimum sum edge coloring of planar bipartite graphs is NP-hard.

List edge coloring is polynomial-time solvable for trees [19]. In fact, for trees the more general problem of list edge multicoloring is polynomial-time solvable as well $[19,21]$. However, by Theorem 3.1 these algorithms cannot be generalized for bounded treewidth graphs, since the problem is NP-complete already for partial 2-trees.

\section{References}

[1] M. Biró, M. Hujter, and Zs. Tuza. Precoloring extension. I. Interval graphs. Discrete Math., 100(1-3):267-279, 1992.

[2] H. L. Bodlaender. A partial $k$-arboretum of graphs with bounded treewidth. Theoret. Comput. Sci., 209(1-2):1-45, 1998.

[3] C. J. Colbourn. The complexity of completing partial Latin squares. Discrete Appl. Math., 8(1):25-30, 1984.

[4] B. Courcelle. The monadic second-order logic of graphs. I. Recognizable sets of finite graphs. Inform. and Comput., 85(1):12-75, 1990.

[5] D. de Werra, M. Demange, B. Escoffier, J. Monnot, and V. T. Paschos. Weighted coloring on planar, bipartite and split graphs: complexity and improved approximation. In ISAAC 2004, 2004.

[6] T. Easton and R. G. Parker. On completing Latin squares. Discrete Appl. Math., 113(2-3):167-181, 2001.

[7] P. Erdős, A. L. Rubin, and H. Taylor. Choosability in graphs. In Proceedings of the West Coast Conference on Combinatorics, Graph Theory and Computing (Humboldt State Univ., Arcata, Calif., 1979), pages 125-157, Winnipeg, Man., 1980. Utilitas Math.

[8] J. Fiala. NP completeness of the edge precoloring extension problem on bipartite graphs. J. Graph Theory, 43(2):156-160, 2003.

[9] T. Fujino, I. Isobe, X. Zhou, and T. Nishizeki. Linear algorithm for finding list edge-colorings of series-parallel graphs. IEICE Trans. Fundamentals, E86-D(No. 2):186-190, 2003.

[10] T. Fujino, X. Zhou, and T. Nishizeki. List edge-colorings of series-parallel graphs. IEICE Trans. Fundamentals, E86-A(No. 5):1034-1045, 2003.

[11] I. Holyer. The NP-completeness of edge-coloring. SIAM J. Comput., 10(4):718-720, Nov. 1981.

[12] M. Hujter and Zs. Tuza. Precoloring extension. II. Graph classes related to bipartite graphs. Acta Mathematica Universitatis Comenianae, 62(1):1-11, 1993. 
[13] M. Hujter and Zs. Tuza. Precoloring extension. III. Classes of perfect graphs. Combin. Probab. Comput., 5(1):35-56, 1996.

[14] K. Jansen and P. Scheffler. Generalized coloring for tree-like graphs. Discrete Appl. Math., 75(2):135-155, 1997.

[15] M. Juvan, B. Mohar, and R. Thomas. List edge-colorings of series-parallel graphs. Electron. J. Combin., 6(1):Research Paper 42,6 pp. (electronic), 1999.

[16] D. König. Über graphen und ihre andwendung auf determinantentheorie und mengenlehre. Math. Ann., 77:453-465, 1916.

[17] J. Kratochvíl. Precoloring extension with fixed color bound. Acta Mathematica Universitatis Comenianae, 62(2):139-153, 1993.

[18] D. Leven and Z. Galil. NP completeness of finding the chromatic index of regular graphs. Journal of Algorithms, 4(1):35-44, Mar. 1983.

[19] O. Marcotte and P. D. Seymour. Extending an edge-coloring. J. Graph Theory, 14(5):565-573, 1990.

[20] D. Marx. Complexity results for minimum sum edge coloring, 2004. Manuscript.

[21] D. Marx. List edge multicoloring in graphs with few cycles. Inform. Process. Lett., 89(2):85-90, 2004.

[22] C. Moore and J. M. Robson. Hard tiling problems with simple tiles. Discrete Comput. Geom., 26(4):573-590, 2001.

[23] T. Nishizeki, J. Vygen, and X. Zhou. The edge-disjoint paths problem is NP-complete for series-parallel graphs. Discrete Appl. Math., 115(1-3):177186, 2001.

[24] C. H. Papadimitriou. Computational complexity. Addison-Wesley Publishing Company, Reading, MA, 1994.

[25] D. P. Sanders and Y. Zhao. Planar graphs of maximum degree seven are class I. J. Combin. Theory Ser. B, 83(2):201-212, 2001.

[26] Zs. Tuza. Graph colorings with local constraints - a survey. Discuss. Math. Graph Theory, 17(2):161-228, 1997.

[27] V. G. Vizing. On an estimate of the chromatic class of a $p$-graph. Diskret. Analiz No., 3:25-30, 1964.

[28] V. G. Vizing. Coloring the vertices of a graph in prescribed colors. Diskret. Analiz, (29 Metody Diskret. Anal. v Teorii Kodov i Shem):3-10, 101, 1976.

[29] J. L. Wu. List-edge coloring of series-parallel graphs. Shandong Daxue Xuebao Ziran Kexue Ban, 35(2):144-149, 2000. (in Chinese). 
[30] X. Zhou and T. Nishizeki. Edge-coloring and $f$-coloring for various classes of graphs. J. Graph Algorithms Appl., 3:no. 1, 18 pp., 1999. 